\title{
A theory of coupled diffusion and large deformation in polymeric gels
}

\author{
Wei Hong ${ }^{1}$, Xuanhe Zhao ${ }^{1}$, Jinxiong Zhou ${ }^{1,2}$, and Zhigang Suo ${ }^{1, *}$ \\ ${ }^{1}$ School of Engineering and Applied Sciences, Harvard University, Cambridge, MA 02138 \\ ${ }^{2}$ School of Aerospace, Xi'an Jiaotong University, Xi'an 710049, People’s Republic of China
}

\begin{abstract}
A large quantity of small molecules may migrate into a network of long polymers, causing the network to swell, forming an aggregate known as a polymeric gel. This paper formulates a theory of the coupled mass transport and large deformation. The free energy of the gel results from two molecular processes: stretching the network, and mixing the network with the small molecules. Both the small molecules and the long polymers are taken to be incompressible, a constraint that we enforce by using a Lagrange multiplier, which coincides with the osmosis pressure or the swelling stress. The gel can undergo large deformation of two modes. The first mode results from the fast process of local rearrangement of molecules, allowing the gel to change shape but not volume. The second mode results from the slow process of long-range migration of the small molecules, allowing the gel to change both shape and volume. We assume that the local rearrangement is instantaneous, and model the long-range migration by assuming that the small molecules diffuse inside the gel. The theory is illustrated with a layer of a gel constrained in its plane and subject to a weight in the normal direction. We also predict the scaling behavior of a gel under a conical indenter.
\end{abstract}

Keywords: polymer; gel; diffusion; large deformation; thermodynamics

*email: suo@seas.harvard.edu 


\section{Introduction}

When a species of long polymers are cross-linked into a three-dimensional network, the resulting material, known as an elastomer, is capable of large and recoverable deformation. Another species of molecules, of a low molecular weight, may act as a solvent. When the elastomer is in contact with the solvent, the small molecules can migrate into the network, forming an aggregate known as a polymeric gel. Inside the gel, the small molecules interact among themselves, and with the long polymers, in the same way as molecules in a liquid. That is, the gel is a condensed matter that fills the space, and the small molecules can readily change neighbors. The cross links prevent the long polymers from dissolving in the solvent; rather, the gel swells and shrinks reversibly as the small molecules migrate in and out.

Polymeric gels are used in diverse technologies, including medical devices (Jagur-Grodzinski, 2006), drug delivery (Duncan, 2003; Fischelghodsian et al., 1988; Jeong, et al., 1997; Langer, 1998), tissue engineering (Luo and Shoichet, 2004; Nowak et al., 2002), and stimuli-sensitive actuators (Beebe et al., 2000; Dong et al., 2006; Sidorenko et al., 2007). These applications aside, we and others are also intrigued by gels as a model system that couples mass transport and large deformation, driven by multiple thermodynamic forces, exhibiting complex behaviors. See an online discussion of the mechanics of gels led by Qi (2007). The present paper aims to develop a field theory of coupled mass transport and large deformation in polymeric gels.

Field theories of mass transport in elastic solids date at least back to Gibbs (1878), who formulated a thermodynamic theory of large deformation of an elastic solid that absorbs a fluid, 
assuming that the solid and the fluid have equilibrated. Biot (1941) combined a similar thermodynamic theory with Darcy's law to model the motion of a fluid in a porous elastic solid. The resulting theory, known as poroelasticity, has been used to analyze phenomena ranging from compaction of soils to deformation of tissues. Rice and Cleary (1976) presented several fundamental solutions in poroelasticity. Larche and Cahn (1985) formulated an analogous theory for diffusion of atoms in an elastic crystal. Lai et al. (1991) included the effects of ions and electric fields, with applications to bones and cartilages. The literature has been reviewed by, among others, Detournay and Cheng (1993), Cowin (1999), Wang (2000), and Coussy (2004).

Several groups have formulated theories specifically to couple mass transport and deformation in gels, invoking various conceptual pictures (e.g., Tanaka and Fillmore, 1979; Durning and Morman, 1993; Dolbow et al., 2004; Tsai et al., 2004; and Li et al., 2007). Both the concepts and the materials are sufficiently complex that ample room exists for additional theoretical work to connect principles of mechanics, thermodynamics and kinetics to experiments and to molecular models.

This paper formulates a field theory in the tradition of Gibbs (1878) and Biot (1941). We phrase the theory in terms of nonequilibrium thermodynamics (e.g., Prigogine, 1967; Coleman and Noll, 1963). Such a theory, however, leaves open the free-energy function and the kinetic law, both being material-specific. We will specify them according to the following qualitative pictures.

We first consider thermodynamics. As the small molecules mix with the long polymers, 
the network swells, so that the configurational entropy of the network decreases, but the configurational entropy of mixture increases. Their compromise tends to equilibrate the gel and the solvent, setting the equilibrium swelling ratio. This part of the theory follows directly that of Flory and Rehner (1943), which we have used in a recent study of dielectric gels (Zhao et al., 2007).

We next consider kinetics. For the network of the long polymers to deform, the small molecules in the gel must change neighbors, a process that is thermally-activated, of the same kind as that in a liquid. Fig. 1 sketches two modes of deformation. The first mode results from local rearrangement of molecules, allowing the gel to change shape but not volume. This mode of deformation occurs over a time scale that is independent of the size of the sample. The local rearrangement is of the same kind as rearranging polymer chains during large deformation of a dry elastomer, a process that gives rise to viscoelasticity. The second mode results from long-range migration of the small molecules, allowing the gel to change both shape and volume. This mode of deformation occurs over a time scale that becomes long when the sample is large. Both modes can give rise to large deformation. Local rearrangement is much faster than long-range migration. In formulating the theory, we will assume that local rearrangement is instantaneous, and model long-range migration as a time-dependent process.

The plan of the paper is as follows. Section 2 outlines the nonequilibrium thermodynamic theory. We then prescribe in Section 3 the free-energy function using the Flory-Huggins theory, and in Section 4 a kinetic law assuming that the small molecules diffuse inside the gel. Section 5 discusses boundary conditions, normalization, and dimensionless 
parameters. Section 6 analyzes a layer of a gel constrained in the transverse directions, and subject to a weight in the normal direction. Section 7 discusses the scaling behavior of a gel under a conical indenter.

\section{A nonequilibrium thermodynamic theory}

Following Gibbs (1878) and Biot (1941), and more directly Suo et al. (2007), Suo (2007) and Zhao et al. (2007), this section presents a field theory of gels. Fig. 2 illustrates two ways of doing work. A weight applies a force $P$, drops by a distance $\delta l$, and does work $P \delta l$. A pump maintains the chemical potential $\mu$ of the small molecules, injects a number $\delta M$ of the small molecules into the gel, and does work $\mu \delta M$. We will use the word "weight" as shorthand for any mechanism that applies an external force, and use the word "pump" as shorthand for any mechanism that injects the small molecules. We next paraphrase these statements in terms of field variables.

A gel is an aggregate of a three-dimensional network of long polymers and a species of small molecules. Imagine that we attach to the network a field of markers. We take the dry network under no mechanical load as the reference state, and name each marker using its coordinate $\mathbf{X}$ in the reference state. The external solvent is taken to be in equilibrium by itself. To maintain a uniform description, we may as well place inside the external solvent an imaginary network, of vanishing elastic stiffness. Thus, volume integrals will extend over both the gel and the external solvent, and surface integrals will extend over the area of all interfaces. Let 
$d V(\mathbf{X})$ be an element of volume, and $N_{K}(\mathbf{X}) d A(\mathbf{X})$ be an element of an interface, where $d A(\mathbf{X})$ is the area of the element, and $N_{K}(\mathbf{X})$ is the unit vector normal to the interface between media labeled as - and + , pointing toward medium + . We will measure both the volume element and the area element in the reference state.

In the current state at time $t$, the marker $\mathbf{X}$ moves to a place with coordinate $\mathbf{x}(\mathbf{X}, t)$. Denote the deformation gradient by

$$
F_{i K}=\frac{\partial x_{i}(\mathbf{X}, t)}{\partial X_{K}}
$$

Imagine that we hang to each marker a weight, which applies an external force to the gel. In the current state, let the force due to the weights on an element of volume be $\mathbf{B}(\mathbf{X}, t) d V(\mathbf{X})$, and on an element of an interface be $\mathbf{T}(\mathbf{X}, t) d A(\mathbf{X})$. Define the nominal stress $s_{i K}(\mathbf{X}, t)$ such that

$$
\int s_{i K} \frac{\partial \xi_{i}}{\partial X_{K}} d V=\int B_{i} \xi_{i} d V+\int T_{i} \xi_{i} d A
$$

holds true for any test function $\xi_{i}(\mathbf{X})$.

The divergence theorem shows that

$$
\int s_{i K} \frac{\partial \xi_{i}}{\partial X_{K}} d V=\int\left(s_{i K}^{-}-s_{i K}^{+}\right) N_{K} \xi_{i} d A-\int \frac{\partial s_{i K}}{\partial X_{K}} \xi_{i} d V
$$

Across the interface, $\xi_{i}(\mathbf{X})$ is assumed to be continuous, but the stress need not be continuous. Insisting that the defining equation (2) for the nominal stress hold true for any $\xi_{i}(\mathbf{X})$, we find that the nominal stress obeys that 


$$
\frac{\partial s_{i K}(\mathbf{X}, t)}{\partial X_{K}}+B_{i}(\mathbf{X}, t)=0
$$

in the volume, and that

$$
\left(s_{i K}^{-}(\mathbf{X}, t)-s_{i K}^{+}(\mathbf{X}, t)\right) N_{K}(\mathbf{X}, t)=T_{i}(\mathbf{X}, t)
$$

on an interface. Equations (4) and (5) are familiar equations of force balance in continuum mechanics.

Imagine that we attach to every marker a pump, which injects the small molecules at the chemical potential $\mu(\mathbf{X}, t)$ into the gel, in the vicinity of the marker. In the current state, let the number of the small molecules per unit time injected into a volume element be $r(\mathbf{X}, t) d V(\mathbf{X})$, and into an interface element be $i(\mathbf{X}, t) d A(\mathbf{X})$. The small molecules also migrate in the gel. In the current state, let $J_{K}(\mathbf{X}, t) N_{K} d A(\mathbf{X})$ be the number of the small molecules per unit time crossing an element of area, and $C(\mathbf{X}, t) d V(\mathbf{X})$ be the number of the small molecules in a volume element. We assume that no chemical reaction occurs, so that the number of small molecules is conserved, namely,

$$
\frac{\partial C(\mathbf{X}, t)}{\partial t}+\frac{\partial J_{K}(\mathbf{X}, t)}{\partial X_{K}}=r(\mathbf{X}, t)
$$

in the volume, and

$$
\left(J_{K}^{+}(\mathbf{X}, t)-J_{K}^{-}(\mathbf{X}, t)\right) N_{K}(\mathbf{X}, t)=i(\mathbf{X}, t)
$$

on an interface.

Multiplying both sides of (6) by an arbitrary test function $\zeta(\mathbf{X})$, integrating over the volume of the gel, and applying the divergence theorem, we obtain that 


$$
\int \frac{\partial C}{\partial t} \zeta d V=\int J_{K} \frac{\partial \zeta}{\partial X_{K}} d V+\int r \zeta d V+\int i \zeta d A
$$

The conservation laws (6) and (7) are equivalent to requiring (8) to hold true for an arbitrary test function $\zeta(\mathbf{X})$.

The field equations, (1), (4), (6), are linear differential equations. We next formulate the structure of material laws, using the approach of nonequilibrium thermodynamics (e.g., Prigogine, 1967; Coleman and Noll, 1963).

When the markers displace at the velocity $\delta \mathbf{x} / \delta t$, the field of weights do work at the rate $\int B_{i} \delta x_{i} / \delta t d V+\int T_{i} \delta x_{i} / \delta t d A$. As the field pumps inject the small molecules into the gel at the rates $r$ and $i$, the pumps do work at the rate $\int \mu r d V+\int \mu i d A$. In the current state, let $W d V(\mathbf{X})$ be the free energy in a volume element. The free-energy density is taken to be a function of the deformation gradient and the nominal concentration, $W(\mathbf{F}, C)$. Associated with small changes, $\delta F_{i K}$ and $\delta C$, the free-energy density changes by

$$
\delta W=\frac{\partial W(\mathbf{F}, C)}{\partial F_{i K}} \delta F_{i K}+\frac{\partial W(\mathbf{F}, C)}{\partial C} \delta C .
$$

The gel, the field of weights, and the field of pumps together form a thermodynamic system. The free energy of the system, $G$, is a sum of the free energy of the gel, and the potential energy of the weights and the pumps. Consequently, associated with the changes, the free energy of the system changes at the rate

$$
\frac{\delta G}{\delta t}=\int \frac{\delta W}{\delta t} d V-\int B_{i} \frac{\delta x_{i}}{\delta t} d V-\int T_{i} \frac{\delta x_{i}}{\delta t} d A-\int \mu r d V-\int \mu i d A
$$

This equation, together with (2), (8) and (9), gives that 


$$
\frac{\delta G}{\delta t}=\int\left(\frac{\partial W}{\partial F_{i K}}-S_{i K}\right) \frac{\delta F_{i K}}{\delta t} d V+\int\left(\frac{\partial W}{\partial C}-\mu\right) r d V+\int\left(\frac{\partial W}{\partial C}-\mu\right) i d A+\int J_{K} \frac{\partial}{\partial X_{K}}\left(\frac{\partial W}{\partial C}\right) d V
$$

Thermodynamics dictates that the free energy of the system should never increase, namely,

$$
\frac{\delta G}{\delta t} \leq 0
$$

This inequality must hold true for any arbitrary $r, i, J_{K}$ and $\delta x_{i} / \delta t$. Consequently, every individual integrand in (11) must either be negative or vanish. Each integral in (11) represents a distinct mechanism of energy dissipation. The first integral is due to local rearrangement of the small molecules, the second and third are due to the field of pumps injecting the small molecules, and the fourth is due to long-range migration of the small molecules.

As discussed in the Introduction, the local rearrangement of molecules is a much faster process than long-range migration. We will take the process of local rearrangement to be instantaneous. That is, we assume local equilibrium by neglecting viscosity associated with local rearrangement of molecules. Consequently, the first integrand in (11) vanishes, leading to

$$
s_{i K}=\frac{\partial W(\mathbf{F}, C)}{\partial F_{i K}} .
$$

We also assume local equilibrium between the small molecules in each pump and those in the gel in the vicinity of the marker. Consequently, the second and the third integrands in (11) vanish, leading to

$$
\mu=\frac{\partial W(\mathbf{F}, C)}{\partial C}
$$

Under the local equilibrium assumptions, the stress equals the derivative of the free energy with respect to the deformation gradient, and the chemical potential of the small molecules injected by the pump equals the free energy change associated with adding one small molecule to the 
vicinity of the marker. Once the free-energy function $W(\mathbf{F}, C)$ is prescribed for a gel, (13) and (14) give the equations of state.

We will model long-range migration of the small molecule as a time-dependent process. To ensure that (12) holds true for any flux, we require that the fourth integrand in (11) be negative-definite. One common way to do this is to adopt a kinetic law

$$
J_{K}=-M_{K L} \frac{\partial \mu(\mathbf{X}, t)}{\partial X_{L}},
$$

such that the mobility $M_{K L}$ is a symmetric and positive-definite tensor. The mobility tensor is a function of the deformation gradient and concentration, $M_{K L}(\mathbf{F}, C)$.

Both the free-energy function $W(\mathbf{F}, C)$ and the mobility tensor $M_{K L}(\mathbf{F}, C)$ should remain invariant when the entire system undergoes a rigid-body rotation in the current state. As is well established in continuum mechanics, this statement of objectivity is equivalent to requiring that $W(\mathbf{F}, C)$ and $M_{K L}(\mathbf{F}, C)$ depend on the deformation gradient only through $F_{i K} F_{i M} \cdot$

When a gel is subject to a sudden change in mechanical load, which is then held constant subsequently, the gel evolves over time. Two limiting states of the gel can be analyzed without invoking the kinetic law. In the short-time limit, the solvent molecules inside the gel do not yet have time to redistribute, but the mechanical equilibrium has already been established. Let $C(\mathbf{X})$ be the known field of concentration at the time when the load changes. The short-time limit is a state governed by the mechanical equilibrium equations (4) and (5), as well as the equation of state (13). This boundary value problem is the same as the residual stress problem in the theory 
of elasticity. The short-time limit corresponds to local rearrangement of molecules, as illustrated in Fig. 1.

In the long-time limit, the gel has reached the equilibrium with both the mechanical load and the external solvent. The free energy of the system has reached the minimum, so that $\delta G / \delta t=0$, and the last integrand in (11) must also vanish. Consequently, the chemical potential $\mu$ is homogeneous throughout the gel, and is prescribed by the external solvent; see Section 5. The long-time limit is a state determined by (4), (5), (13) and (14). Because $\mu$ is a known constant, one can solve $C$ from (14) and insert $C$ into (13). This boundary value problem is again the same as the residual stress problem in the theory of elasticity.

\section{Molecular incompressibility and the free-energy function of a model gel}

Under most types of load, the long polymers and the small molecules can undergo large configurational change without appreciable volumetric change. Following a common practice, we assume that the individual long polymers and the individual small molecules are incompressible. Furthermore, the gel is a condensed matter with negligible void space, so that we express the condition of molecular incompressibility as

$$
1+v C=\operatorname{det}(\mathbf{F})
$$

where $v$ is the volume per small molecule, and $v C$ is the volume of the small molecules in the gel divided by the volume of the dry polymers.

The condition of molecular incompressibility can be enforced as a constraint by adding a 
term $\int \Pi(1+v C-\operatorname{det} \mathbf{F}) d V$ to the free energy $G$ of the system, where $\Pi(\mathbf{X}, t)$ is a field of Lagrange multiplier. Recall an algebraic identity, $\partial \operatorname{det}(\mathbf{F}) / \partial F_{i K}=H_{i K} \operatorname{det}(\mathbf{F})$, where $H_{i K}$ is the transpose of the inverse of the deformation gradient, namely, $H_{i K} F_{i L}=\delta_{K L}$ and $H_{i K} F_{j K}=\delta_{i j}$. Consequently, (13) and (14) become

$$
\begin{gathered}
s_{i K}=\frac{\partial W(\mathbf{F}, C)}{\partial F_{i K}}-\Pi H_{i K} \operatorname{det}(\mathbf{F}), \\
\mu=\frac{\partial W(\mathbf{F}, C)}{\partial C}+\Pi v .
\end{gathered}
$$

The small molecules can freely enter or leave the gel, but the long polymers are cross-linked into the network and cannot leave the gel. The situation is analogous to that of a membrane permeable to the solvent but not to the solute. If such a membrane separates a pure solvent and a solution, the solvent will diffuse across the membrane until the solution builds up a pressure, known as the osmotic pressure. From (18), we can interpret the Lagrange multiplier $\Pi$ as the osmotic pressure, which increases the chemical potential for the solvent to enter the gel.

Recalling that the true stress relates to the nominal stress by $\sigma_{i j}=s_{i K} F_{j K} / \operatorname{det}(\mathbf{F})$, we rewrite (17) as

$$
\sigma_{i j}=\frac{\partial W(\mathbf{F}, C)}{\partial F_{i K}} \frac{F_{j K}}{\operatorname{det}(\mathbf{F})}-\Pi \delta_{i j} .
$$

The first term is the elastic stress due to stretching the network of the long polymers, while the second term is the osmotic pressure. When the gel is subject to no weights, but is in equilibrium with the solvent, the stresses vanish, $\sigma_{i j}=0$. Under this condition, the elastic stress must balance the osmotic pressure. As the small molecules migrate into the network, the osmotic pressure causes the gel to swell against the elastic stress. Consequently, the osmosis 
pressure is also called the swelling stress. When the field in a gel is inhomogeneous, the osmosis pressure $\Pi$ is also an inhomogeneous field, which can be solved as a part of an initial and boundary problem.

To solve initial and boundary value problems, we need an explicit form of the free-energy function, $W(\mathbf{F}, C)$. Our intention is to illustrate the general procedure, and to describe approximate behavior of polymeric gels. As such, we will adopt a simplest form of the free-energy function. To describe accurate behavior of a specific gel would require adding terms and parameters to fit experimental data, a task that is beyond the scope of this paper.

The free energy of a polymeric gel comes from two molecular processes: stretching the network of the polymers, and mixing the polymers and the small molecules. Following Flory and Rehner (1943), we assume that the free energy of the gel takes the form

$$
W(\mathbf{F}, C)=W_{s}(\mathbf{F})+W_{m}(C),
$$

where $W_{s}$ and $W_{m}$ are the contributions from stretching and mixing, respectively.

Let $\lambda_{1}, \lambda_{2}$ and $\lambda_{3}$ be the three stretches. The free energy due to stretching a network of polymers is taken to be (Flory, 1953)

$$
W_{s}(\mathbf{F})=\frac{1}{2} N k T\left(\lambda_{1}^{2}+\lambda_{2}^{2}+\lambda_{3}^{2}-3-2 \log \lambda_{1} \lambda_{2} \lambda_{3}\right),
$$

where $N$ is the number of polymer chains in the gel divided by the volume of the dry polymers, and $k T$ is the temperature in the unit of energy. Other forms of the free energy of stretching are available and may be used to fit experimental data (see reviews by Boyce and Arruda, 2000; Marckmann and Verron, 2006; Horkay and McKenna, 2007). 
When the long polymers are not cross-linked, the long polymers and the small molecules form a liquid solution. The free energy of mixing is taken to be (Flory, 1942; Huggins, 1941)

$$
W_{m}(C)=-\frac{k T}{v}\left[v C \log \left(1+\frac{1}{v C}\right)+\frac{\chi}{1+v C}\right] .
$$

The first term inside of the bracket comes from the entropy of mixing, and the second from the enthalpy of mixing, where $\chi$ is a dimensionless parameter. The enthalpy of mixing motivates the small molecules to enter the gel if $\chi<0$, but motivates the small molecules to leave the gel if $\chi>0$.

Let $s_{1}, s_{2}$ and $s_{3}$ be the three principal nominal stresses. Equation (17) is specialized to

$$
\begin{aligned}
& s_{1}=N k T\left(\lambda_{1}-\lambda_{1}^{-1}\right)-\Pi \lambda_{2} \lambda_{3}, \\
& s_{2}=N k T\left(\lambda_{2}-\lambda_{2}^{-1}\right)-\Pi \lambda_{3} \lambda_{1}, \\
& s_{3}=N k T\left(\lambda_{3}-\lambda_{3}^{-1}\right)-\Pi \lambda_{1} \lambda_{2},
\end{aligned}
$$

Equation (18) is specialized to

$$
\mu=k T\left[\log \frac{v C}{1+v C}+\frac{1}{1+v C}+\frac{\chi}{(1+v C)^{2}}\right]+\Pi v .
$$

Equations (23) and (24) constitute the equations of state for the model gel.

\section{Kinetic law}

Following Biot (1941), much of the literature on poroelasticity has modeled the passage of a fluid in a porous elastic solid by using Darcy's law. However, we find it inappropriate to use Darcy's law for polymeric gels. Darcy's law pictures a fluid flowing in pores inside the 
solid, with the permeability being proportional to the square of the pore size. In a swollen gel, however, the major part of the space is occupied by the small molecules. When the small molecules move to allow the network to deform, the resistance to their motion comes mostly from other small molecules, much like the resistance felt by a tracer molecule diffusing in a liquid. To the first approximation, the size of the cell in the network is irrelevant to the coefficient of diffusion of the small molecules.

Tanaka et al. (1973) and Tanaka and Fillmore (1979) developed a kinetic theory by assuming that swelling is rate-limited by the friction between the polymers and liquid. They modeled friction as a body force proportional to the velocity of the network. This theory forbids the gel to deform quickly under a sudden change in mechanical load. The theory has also not been coupled to large deformation.

As discussed in the Introduction, we will develop a kinetic law by assuming that the small molecules diffuse in the gel. Diffusion has long been used to model phenomena such as creep in polycrystalline solids (Herring, 1950), migration of atoms in elastic crystals (Larche and Cahn, 1985), and migration of small molecules in gels (Buckley and Berger, 1962). As a simplest model, we assume that the coefficient of diffusion of the solvent molecules, $D$, is isotropic and is independent of deformation gradient and concentration. This simplification is reasonable for a swollen gel, in which the small molecules are the majority component. Furthermore, the local behavior of a molecular group on a freely-jointed long polymer chain is quite similar to a small molecule. Let $c(\mathbf{X}, t)$ be the number of the small molecules per unit 
volume in the current state, $j_{i}(\mathbf{X}, t)$ be the number of the small molecules per unit time crossing per unit area in the current state, and $\mu(\mathbf{X}, t)$ be the chemical potential. The flux relates to the gradient of the chemical potential by a well known equation (e.g., Feynman et al., 1963)

$$
j_{i}=-\frac{c D}{k T} \frac{\partial \mu}{\partial x_{i}}
$$

Although written in terms of the true quantities, this equation is by no means an Eulerian description; rather, it is an updated Lagrangian description, with $c, j_{i}$ and $\mu$ being quantities associated with the markers.

We next convert (25) into an expression using the nominal quantities, in the form of (15). The true concentration relates to the nominal concentration as

$$
c=\frac{C}{\operatorname{det}(\mathbf{F})}
$$

A material element of area is $N_{K} d A$ in the reference state and deforms to $n_{i} d a$ in the current state. Recall an identity, $\operatorname{det}(\mathbf{F}) N_{K} d A=F_{i K} n_{i} d a$. The number of molecules crossing the material element per unit time can be written in two equivalent ways:

$$
j_{i} n_{i} d a=J_{K} N_{K} d A .
$$

Consequently, the true flux relates to the nominal flux as

$$
j_{i}=\frac{F_{i K}}{\operatorname{det}(\mathbf{F})} J_{K}
$$

Using the chain rule of partial derivatives, we obtain that 


$$
\frac{\partial \mu}{\partial X_{K}}=\frac{\partial \mu}{\partial x_{i}} F_{i K}
$$

Comparing (15) and (25), and invoking the condition of molecular incompressibility (16), we relate the mobility tensor to the coefficient of diffusion:

$$
M_{K L}=\frac{D}{v k T} H_{i K} H_{i L}[\operatorname{det}(\mathbf{F})-1]
$$

When deformation is large, the mobility tensor as defined using nominal quantities is anisotropic.

\section{Boundary conditions, normalizations and dimensionless parameters}

Outside the gel, the solvent is composed of a species of small molecules, either in a liquid or in a gaseous state. The reference state of the solvent is set to be a liquid in equilibrium with its own vapor. Let $p_{0}$ be the vapor pressure, and $p$ be the pressure in the solvent in the current state. In the current state, the chemical potential of the small molecules in the external solvent, $\mu_{\text {ext }}$, is the change in the free energy associated with relocating one small molecule from the reference state to the current state. If the solvent in the current state is a gas $\left(p<p_{0}\right)$, and is modeled as an ideal gas, the chemical potential is $\mu_{\text {ext }}=k T \log \left(p / p_{0}\right)$. If the solvent in the current state is a liquid ( $p>p_{0}$ ), and the molecules are taken to be incompressible, the chemical potential is $\mu_{e x t}=v\left(p-p_{0}\right)$.

Consider a gel and an external liquid solvent both subject to a state of homogeneous hydrostatic stress. Under the condition of molecular incompressibility, the volume of a small 
molecule does not change when it relocates from the external liquid to the gel. Consequently, the homogeneous hydrostatic stress applied on both the gel and the external liquid does not affect the thermodynamic state of the system. We should emphasize that this conclusion only applies when the external solvent is liquid. If the external solvent is gaseous, however, the hydrostatic stress does do work when a small molecule relocates from the gas to the gel.

For an initial value problem, we need to prescribe boundary conditions on an interface between a gel and a solvent. The chemical boundary condition can be prescribed by giving the boundary value of either the flux $i$ or the chemical potential $\mu$. The mechanical boundary condition can be prescribed by giving the boundary value of either the position $\mathbf{x}$ or the traction $\mathbf{T}$.

We will normalize the chemical potential by $k T$, and normalize the stresses by $k T / v$. A representative value of the volume per molecule is $v=10^{-28} \mathrm{~m}^{3}$. At room temperature, $k T=4 \times 10^{-21} \mathrm{~J}$ and $k T / v=4 \times 10^{7} \mathrm{~Pa}$.

The free-energy functions (21) and (22) introduce two dimensionless material parameters: $N v$ and $\chi$. In the absence of small molecules, the cross-linked polymers have a shear modulus $N k T$ under the small-strain conditions, with the representative values $N k T=10^{4} \sim 10^{7}$ $\mathrm{N} / \mathrm{m}^{2}$, which gives the range $N v=10^{-4} \sim 10^{-1}$. The parameter $\chi$ is a dimensionless measure of the enthalpy of mixing, with representative values $\chi=0 \sim 1.2$. For applications that prefer gels with large swelling ratios, materials with low $\chi$ values are used. In the numerical examples below, we will take the values $N v=10^{-3}$ and $\chi=0.2$. 
The theory does not have an intrinsic time scale or an intrinsic length scale. However, if a boundary value problem has a length scale, say the characteristic size of the sample, $L$, the diffusion time scale is $L^{2} / D$, which we will use to normalize time. The coefficient of diffusion of a particle in a liquid relates to the viscosity of the liquid by the Stokes-Einstein formula: $D=k T /(6 \pi R \eta)$, where $R$ is the radius of the particle. Although this formula was derived for particles much larger than molecules, we will use the formula to estimate the order of magnitude of the coefficient of self-diffusion. For example, for water at room temperature, taking $R=3 \times 10^{-10} \mathrm{~m}$ and $\eta=0.89 \times 10^{-3} \mathrm{~Pa} \cdot \mathrm{s}$, we obtain that $D=8 \times 10^{-10} \mathrm{~m}^{2} / \mathrm{s}$, an order of magnitude that is comparable to the coefficient of diffusion of many different molecules in water. For a size scale $L=10^{-3} \mathrm{~m}$, we find that the time scale is $L^{2} / D=10^{3} \mathrm{~s}$.

\section{Uniaxial creep}

Fig. 3 sketches a layer of a gel immersed in a liquid solvent. The gel first swells without constraint to equilibrate with the external solvent. The swollen gel is then bonded to a rigid substrate and, at time zero, a weight is applied on the top surface of the gel via a permeable plate. The solvent molecules can diffuse out of the gel through the plate, and the gel thins down gradually. This initial value problem was first analyzed by Biot (1941) within his theory of linear poroelasticity, and is now analyzed within the nonlinear theory developed in this paper.

Let $L$ be the thickness of the dry polymer, and $s$ be the applied weight divided by the area of the dry polymer. The chemical potential of the external solvent is set to be zero. The 
vapor pressure $p_{0}$ is taken to be small compared to $s$, and is neglected in the following analysis. Let $X_{1}$ and $X_{2}$ be the material coordinates in the plane of the layer, and $X_{3}$ be the material coordinate normal to the layer and pointing downwards. The substrate keeps the lateral stretches $\lambda_{1}=\lambda_{2}$ constant. At time $t$, the marker $X_{3}$ moves to a place $x_{3}\left(X_{3}, t\right)$. The vertical stretch is

$$
\lambda_{3}\left(X_{3}, t\right)=\frac{\partial x_{3}\left(X_{3}, t\right)}{\partial X_{3}}
$$

The stretch is inhomogeneous and evolves with time.

We first consider the two limiting states. Setting $s_{3}=s$ in (23c) and $\mu=0$ in (24), and eliminating $\Pi$, we obtain that

$$
\frac{s v}{k T}=N v\left(\lambda_{3}-\lambda_{3}^{-1}\right)+\lambda_{1}^{2} \log \left(1-\frac{1}{\lambda_{3} \lambda_{1}^{2}}\right)+\frac{1}{\lambda_{3}}+\frac{\chi}{\lambda_{3}^{2} \lambda_{1}^{2}} .
$$

When the gel swells under no constraint, $s=0$ and $\lambda_{1}=\lambda_{2}=\lambda_{3}$. Solving (32), we obtain the equilibrium swelling ratio under no constraint, $\lambda_{1}=\lambda_{2}=\lambda_{3}=3.215$. Subsequently, the gel is bonded to the rigid substrate, which keeps $\lambda_{1}=\lambda_{2}=3.215$ constant. Right after the weight is applied on the top of the gel, $t=0^{+}$, the small molecules do not have time to diffuse and the gel behaves like an incompressible elastic body. Since the lateral stretches are held constant, the vertical stretch must also remain unchanged, $\lambda_{3}\left(X_{3}, 0^{+}\right)=3.215$. After a long time, $t \rightarrow \infty$, the gel establishes a new equilibrium with both the weight and the external liquid. The vertical stretch $\lambda_{3}\left(X_{3}, \infty\right)$ can be obtained by solving Eq. (32) for any given value of stress $s$. 
As time progresses, the stretch in the gel gradually changes from $\lambda_{3}\left(X_{3}, 0^{+}\right)=3.215$ to $\lambda_{3}\left(X_{3}, \infty\right)$, and the change propagates from the surface toward deep inside the gel. We next analyze the evolution process. Equation (23c) is specialized to

$$
s_{3}\left(X_{3}, t\right)=\frac{\partial W_{s}}{\partial \lambda_{3}}-\lambda_{1}^{2} \Pi\left(X_{3}, t\right) .
$$

Equation (24) becomes

$$
\mu\left(X_{3}, t\right)=\frac{d W_{m}}{d C}+v \Pi\left(X_{3}, t\right)
$$

The mechanical equilibrium equations (4) and (5) are satisfied if $s_{3}\left(X_{3}, t\right)=s$ throughout the gel. The chemical potential, however, is inhomogeneous in the gel and evolves with time. The condition of molecular incompressibility becomes

$$
1+v C=\lambda_{1}^{2} \lambda_{3}
$$

The kinetic law (15) is specialized to

$$
J\left(X_{3}, t\right)=-\frac{D\left(\lambda_{1}^{2} \lambda_{3}-1\right)}{v k T \lambda_{3}^{2}} \frac{\partial \mu\left(X_{3}, t\right)}{\partial X_{3}} .
$$

No pumps exist inside the gel, so that the conservation of solvent molecules is expressed as

$$
\frac{\partial C\left(X_{3} t\right)}{\partial t}=-\frac{\partial J\left(X_{3} t\right)}{\partial X_{3}}
$$

The boundary conditions are as follows. The chemical potential at the surface of the gel equals that in the liquid, $\mu(0, t)=0$. The rigid substrate is taken to be impermeable to the solvent, $J(L, t)=0$.

Substituting Eqs. (33)-(36) into (37), we arrive at a partial differential equation for the 
function $\lambda_{3}\left(X_{3}, t\right)$ :

$$
\frac{\partial \lambda_{3}}{\partial t}=D \frac{\partial}{\partial X_{3}}\left[\left(1-\frac{1}{\lambda_{1}^{2} \lambda_{3}}\right)\left(\frac{1}{\left(\lambda_{1}^{2} \lambda_{3}-1\right) \lambda_{1}^{2} \lambda_{3}^{3}}-\frac{2 \chi}{\lambda_{1}^{4} \lambda_{3}^{4}}+\frac{N v}{\lambda_{1}^{2} \lambda_{3}}\left(1+\frac{1}{\lambda_{3}^{2}}\right)\right) \frac{\partial \lambda_{3}}{\partial X_{3}}\right] .
$$

The boundary conditions become

$$
\begin{gathered}
\lambda_{1}^{2}\left[\log \left(1-\frac{1}{\lambda_{3} \lambda_{1}^{2}}\right)+\frac{1}{\lambda_{3} \lambda_{1}^{2}}+\frac{\chi}{\lambda_{3}^{2} \lambda_{1}^{4}}+\frac{N v}{\lambda_{1}^{2}}\left(\lambda_{3}-\frac{1}{\lambda_{3}}\right)\right]_{X_{3}=0}=\frac{s v}{k T}, \\
\frac{\partial \lambda_{3}(L, t)}{\partial X_{3}}=0 .
\end{gathered}
$$

The initial condition is

$$
\lambda_{3}\left(X_{3}, 0\right)=3.215 \text {. }
$$

The partial differential equation is solved numerically.

Fig. 4 plots the function $\lambda_{3}\left(X_{3}, t\right)$ for a gel subject to a weight at the level $s v / k T=-0.05$. The surface of the gel $\left(X_{3}=0\right)$ is assumed to equilibrate with the solvent instantaneously and at all times, so that the stretch on the surface is always at the long-time limit. Initially, the stretch inside the gel is unperturbed and remains at the short-time limit. As time progresses, the stretch evolves from the short-time limit to the long-time limit, and the change propagates gradually into the depth of the gel.

Fig. 5 plots the thickness of the gel as a function of time at several levels of the applied weight. The horizontal axis is $\sqrt{t D / L^{2}}$. Initially, the stretch of the gel in the thickness direction coincides with that of free swelling $l / L=3.215$. The rate process may be divided into two stages. When time is short, $t<<L^{2} / D$, only the molecules near the top surface diffuse appreciably, so that the thickness of the gel, $L$, does not affect the rate of diffusion. 
Consequently, the problem has no length scale, and the stretch $\lambda_{3}\left(X_{3}, t\right)$ evolves by a self-similar profile (see below), so that the reduction in thickness scales linearly in $\sqrt{D t}$. When time is long, $t>>L^{2} / D$, the thickness approaches a constant, corresponding to the long-time limit.

We now examine the self-similar solution for an infinitely thick layer. The initial value problem has no length scale, so that the profile of the stretch must take the form

$$
\lambda_{3}\left(X_{3}, t\right)=\lambda_{3}(\xi), \quad \text { with } \xi=X_{3} / \sqrt{D t} .
$$

Consequently, (38) becomes an ordinary differential equation:

$$
\begin{aligned}
& \left(1-\frac{1}{\lambda_{1}^{2} \lambda_{3}}\right)\left[\frac{1}{\left(\lambda_{1}^{2} \lambda_{3}-1\right) \lambda_{1}^{2} \lambda_{3}^{3}}-\frac{2 \chi}{\lambda_{3}^{4} \lambda_{1}^{4}}+\frac{N v}{\lambda_{3} \lambda_{1}^{2}}\left(1+\frac{1}{\lambda_{3}^{2}}\right)\right] \frac{d^{2} \lambda_{3}}{d \xi^{2}}+\frac{\xi}{2} \frac{d \lambda_{3}}{d \xi} \\
& +\left[-\frac{4}{\lambda_{1}^{4} \lambda_{3}^{5}}+2 \chi \frac{4 \lambda_{1}^{2} \lambda_{3}-5}{\lambda_{1}^{6} \lambda_{3}^{6}}+\frac{N v}{\lambda_{1}^{4} \lambda_{3}^{5}}\left(4-3 \lambda_{1}^{2} \lambda_{3}+2 \lambda_{3}^{2}-\lambda_{1}^{2} \lambda_{3}^{3}\right)\right]\left(\frac{d \lambda_{3}}{d \xi}\right)^{2}=0
\end{aligned}
$$

This equation is integrated numerically. Fig. 6 plots the function $\lambda_{3}(\xi)$ for several levels of the applied weight. The layer may be divided into two regions by a diffusion front. Behind the front, the gel approaches the long-time limit. Ahead of the front, the gel approaches the short-time limit. The coordinate of the front scales with $\sqrt{D t}$.

\section{Scaling behavior of a gel under a conical indenter}

Indentation has long been used to characterize materials. Following an approach of Cheng and Cheng (1998), here we study the scaling behavior of a gel under a conical indenter. The gel is first equilibrated to a solvent held at the chemical potential $\mu_{\text {ext }}$. A rigid indenter, of 
a conical shape with the half angle $\theta$, is then indented vertically into the gel. Let $P$ be the force applied to the indenter, and $h$ be the depth of the indentation. If $h$ is much smaller than the size of the gel, then $h$ is the only length scale in the problem. Imagine that we indent at time $t=0$ and hold the depth $h$ constant. The force $P$ will first jump to the short-time limit, and then gradually relax to the long-time limit. Dimensional considerations dictate that

$$
P=h^{2} \frac{k T}{v} f\left(\frac{t}{h^{2} / D}\right) .
$$

The dimensionless function $f$ also depends on other dimensionless parameters in the problem, namely, the material parameters $N v$ and $\chi$, the half angle $\theta$ of the conical indenter, and the chemical potential of the solvent $\mu_{\text {ext }} / k T$. These parameters, however, remain constant during indentation. Both the short-time limit $P_{\text {short }}$ and the long-time limit $P_{\text {long }}$ scales as $h^{2} k T / v$. Fig. 7a sketches these parabolic scaling laws, $P_{\text {short }} \propto h^{2}$ and $P_{\text {long }} \propto h^{2}$. A vertical line in Fig. 7a represents a relaxation experiment, in which the depth $h$ is held constant, while the force on the indenter decreases over time (Fig. 7b).

As indicated in Fig. 7b, we define a characteristic time for the relaxation experiment by the time needed for the force to reduce to the mid-point, $\left(P_{\text {short }}+P_{\text {long }}\right) / 2$. This characteristic time scales as

$$
t_{1 / 2}^{\text {relax }}=\frac{h^{2}}{D} k
$$

where the dimensionless factor $k$ depends on the dimensionless parameters identified above.

One can also conduct a creep test by holding the force $P$ constant, and record the depth $h$ as a function of time. A dimensional analysis of our theory shows that 


$$
h=\sqrt{\frac{P v}{k T}} F\left(\frac{t}{P v / k T D}\right),
$$

where the dimensionless function $F$ also depends on other dimensionless parameters identified above. The short- and the long-time limits are still given by the two curves in Fig. 7a, where the creep test is indicated by the horizontal line. Fig. 7c sketches the indentation depth as a function of time in the creep test, where the characteristic time for the creep experiment is

defined by the time needed for the depth to increase to the mid-point, $\left(h_{\text {short }}+h_{\text {long }}\right) / 2$. This characteristic time scale as

$$
t_{1 / 2}^{\text {creep }}=\frac{P V}{k T D} K
$$

where the dimensionless factor $K$ depends on the dimensionless parameters identified above.

\section{Concluding remarks}

We have presented a theory of coupled diffusion and large deformation within the framework of nonequilibrium thermodynamics. All fields are given as functions of the material coordinates, so that the domain of the problem remains unchanged as time increases. The theory consists of linear partial differential equations corresponding to force balance and conservation of the number of molecules. Nonlinearity is introduced in material-specific functions: the free-energy function $W(\mathbf{F}, C)$ and the mobility tensor $M_{K L}(\mathbf{F}, C)$. They can be related to experimental data and to molecular theories. In particular, we have developed a kinetic law by assuming that the small molecules diffuse in the gel. Basic solutions, such as a 
layer of a gel under a weight, and a block of a gel under a conical indenter, will be useful to characterize materials.

\section{Acknowledgements}

This research was supported by the Army Research Office through contract W911NF-04-1-0170, and by the National Science Foundation through the MRSEC at Harvard University. Visits of ZS to the Institute of Process Engineering, of Chinese Academy of

Sciences, have been supported by a project entitled Research Collaboration on Multi-Scale Science of Complex Systems. 


\section{References}

Beebe, D.J., Moore, J.S., Bauer, J.M., Yu, Q., Liu, R.H., Devadoss, C., Jo, B.H., 2000. Functional hydrogel structures for autonomous flow control inside microfluidic channels. Nature 404 (6778), 588.

Biot, M.A., 1941. General theory of three-dimensional consolidation. Journal of Applied Physics 12 (2), 155-164.

Boyce, M.C. and E.M. Arruda, 2000. Constitutive models of rubber elasticity: A review. Rubber Chemistry and Technology 73, 504-523

Buckley, D.J., Berger, M., 1962. Swelling of Polymer Systems in Solvents .2. Mathematics of Diffusion. Journal of Polymer Science 56 (163), 175-188.

Cheng, Y.T. and Cheng, C.M., 1998. Scaling approach to conical indentation in elastic-plastic solids with work hardening. J. Appl. Phys. 84, 1284-1291.

Coleman, B.D. and Noll, W., 1963. The thermodynamics of elastic materials with heat conduction and viscosity. Archive for Rational Mechanics and Analysis 13, 167-178.

Coussy, O., 2004. Poromechanics, Wiley, NJ.

Cowin, S.C., 1999. Bone poroelasticity. J. Biomechanics 32, 217-238.

Detournay, E., and Cheng, A.H.-D., 1993. Fundamentals of poroelasticity. In Comprehensive Rock Engineering: Principles, Practices and Projects, Vol. 2, ed. J.A. Hudson, pp. 113-171. Pergamon Press, Oxford, UK.

Dolbow, J., Fried, E., Jia, H.D., 2004. Chemically induced swelling of hydrogels. Journal of the Mechanics and Physics of Solids 52 (1), 51-84.

Dong, L., Agarwal, A.K., Beebe, D.J., Jiang, H.R., 2006. Adaptive liquid microlenses activated by stimuli-responsive hydrogels. Nature 442 (7102), 551-554.

Duncan, R., 2003. The dawning era of polymer therapeutics. Nature Reviews Drug Discovery 2 (5), 347-360.

Durning, C.J., Morman, K.N., 1993. Nonlinear Swelling of Polymer Gels. Journal of Chemical Physics 98 (5), 4275-4293.

Feynman, R.P., Leighton, R.B., and Sands, M., 1963. The Feynman Lectures on Physics, p. I-43-9.

Fischelghodsian, F., Brown, L., Mathiowitz, E., Brandenburg, D., Langer, R., 1988. Enzymatically Controlled Drug Delivery. Proceedings of the National Academy of Sciences of the United States of America 85 (7), 2403-2406.

Flory, P.I., 1942. Thermodynamics of high polymer solutions. Journal of Chemical Physics 10 (1), 51-61.

Flory, P.J., 1953. Principles of Polymer Chemistry. Cornell University Press, Ithaca.

Flory, P.J., Rehner, J., 1943. Statistical mechanics of cross-linked polymer networks II Swelling. Journal of Chemical Physics 11 (11), 521-526.

Gibbs, J.W., 1878. pages 184, 201, 215 in The Scientific Papers of J. Willard Gibbs. Digital copy of the book is freely available at http://books.google.com/.

Herring, C., 1950, Diffusional Viscosity of a Polycrystalline Solid. J. Appl. Phys. 21, 437-445. 
Horkay, F. and McKenna, G.B., 2007. Polymer networks and gels. pp. 497-523 in Physical properties of Polymers Handbook. edited by J.E. Mark. Springer, New York.

Huggins, M.L., 1941. Solutions of long chain compounds. Journal of Chemical Physics 9 (5), 440-440.

Jagur-Grodzinski, J., 2006. Polymers for tissue engineering, medical devices, and regenerative medicine. Concise general review of recent studies. Polymers for Advanced Technologies 17 (6), 395-418.

Jeong, B., Bae, Y.H., Lee, D.S., Kim, S.W., 1997. Biodegradable block copolymers as injectable drug-delivery systems. Nature 388 (6645), 860-862.

Lai, W.M., Hou, J.S., Mow, V.C., 1991. A Triphasic Theory for the Swelling and Deformation Behaviors of Articular-Cartilage. Journal of Biomechanical Engineering-Transactions of the Asme 113 (3), 245-258.

Langer, R., 1998. Drug delivery and targeting. Nature 392 (6679), 5-10.

Larche, F.C., Cahn, J.W., 1985. The Interactions of Composition and Stress in Crystalline Solids. Acta Metallurgica 33 (3), 331-357.

Li, H., Luo, R., Birgersson, E., Lam, K.Y., 2007a. Modeling of multiphase smart hydrogels responding to $\mathrm{pH}$ and electric voltage coupled stimuli. Journal of Applied Physics 101 (11).

Luo, Y., Shoichet, M.S., 2004. A photolabile hydrogel for guided three-dimensional cell growth and migration. Nature Materials 3 (4), 249-253.

Marckmann, G. and Verron, E., 2006. Comparison of hyperelastic models for rubberlike materials, Rubber Chemistry and Technology 79, 835-858.

Nowak, A.P., Breedveld, V., Pakstis, L., Ozbas, B., Pine, D.J., Pochan, D., Deming, T.J., 2002. Rapidly recovering hydrogel scaffolds from self-assembling diblock copolypeptide amphiphiles. Nature 417 (6887), 424-428.

Prigogine, I., 1967. Introduction to Thermodynamics of Irreversible Processes, $3^{\text {rd }}$ ed., Wiley, New York.

Rice, J.R., Cleary, M.P., 1976. Some Basic Stress Diffusion Solutions for Fluid-Saturated Elastic Porous-Media with Compressible Constituents. Reviews of Geophysics 14 (2), 227-241.

Sidorenko, A., Krupenkin, T., Taylor, A., Fratzl, P., Aizenberg, J., 2007. Reversible switching of hydrogel-actuated nanostructures into complex micropatterns. Science 315 (5811), 487-490.

Suo, Z., 2007. Poroelasticity, or diffusion in an elastic solid. http://imechanica.org/node/987

Suo, Z., Zhao, X., Greene, W.H., 2007. A nonlinear field theory of deformable dielectrics. Journal of Mechanics and Physics of Solids, doi:10.1016/j.jmps.2007.1005.1021.

Qi, H.J., 2007. The mechanics of hydrogel, http://imechanica.org/node/1641.

Tanaka, T., Fillmore, D.J., 1979. Kinetics of Swelling of Gels. Journal of Chemical Physics 70 (3), 1214-1218.

Tanaka, T., Hocker, L.O., Benedek, G.B., 1973. Spectrum of Light Scattered from a Viscoelastic Gel. Journal of Chemical Physics 59 (9), 5151-5159.

Tsai, H., Pence, T.J., Kirkinis, E., 2004. Swelling induced finite strain flexure in a rectangular block of an isotropic elastic material. Journal of Elasticity 75 (1), 69-89. 
Wang, H.F., 2000. Theory of Linear Poroelasticity. Princeton University Press.

Zhao, X., Hong, W., Suo, Z., 2007. Stretching and polarizing a dielectric gel immersed in a solvent. http://imechanica.org/node/1861. 


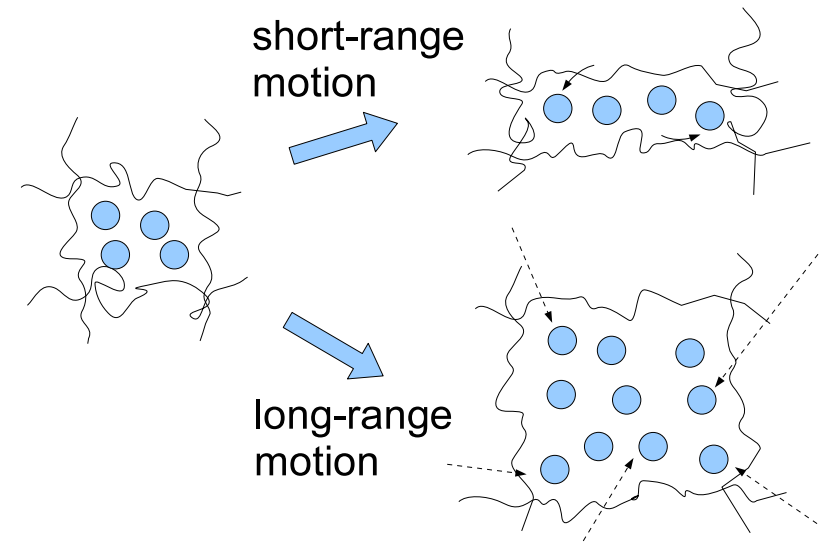

Figure 1. A schematic of two modes of deformation in a gel consisting of a network of long polymers (lines) and a species of small molecules (dots). The short-range motion locally rearranges the small molecules, so that the gel can change shape but not the volume. The long-range motion does not conserve the number of small molecules locally, so that the gel can change both shape and volume. 


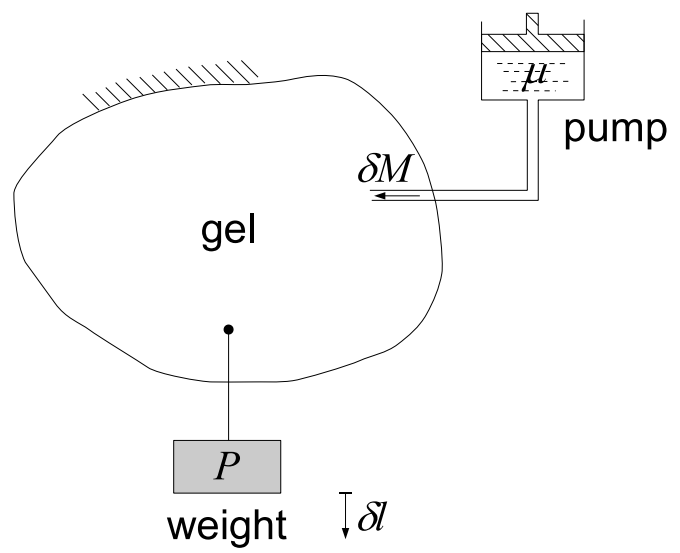

Figure 2. A mechanical load is applied by hanging a weight to the network. A chemical load is applied by using a pump to inject small molecules into the gel. 


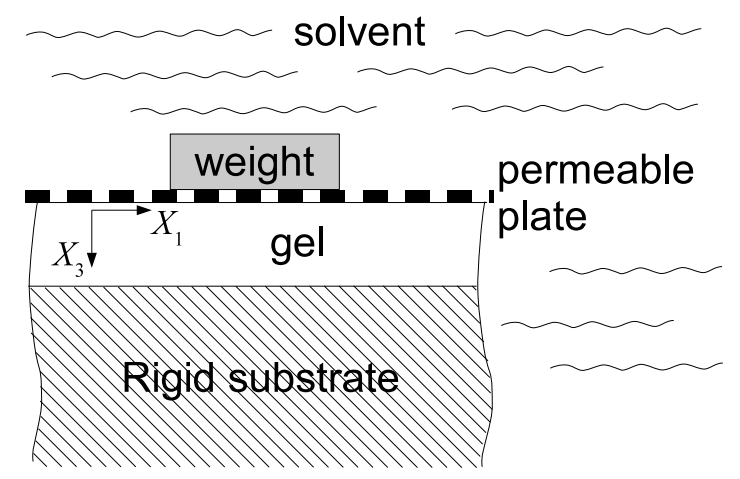

Figure 3. A gel is bonded to a rigid substrate, immersed in a solvent, and subject to an applied weight via a plate permeable to the small molecules. 


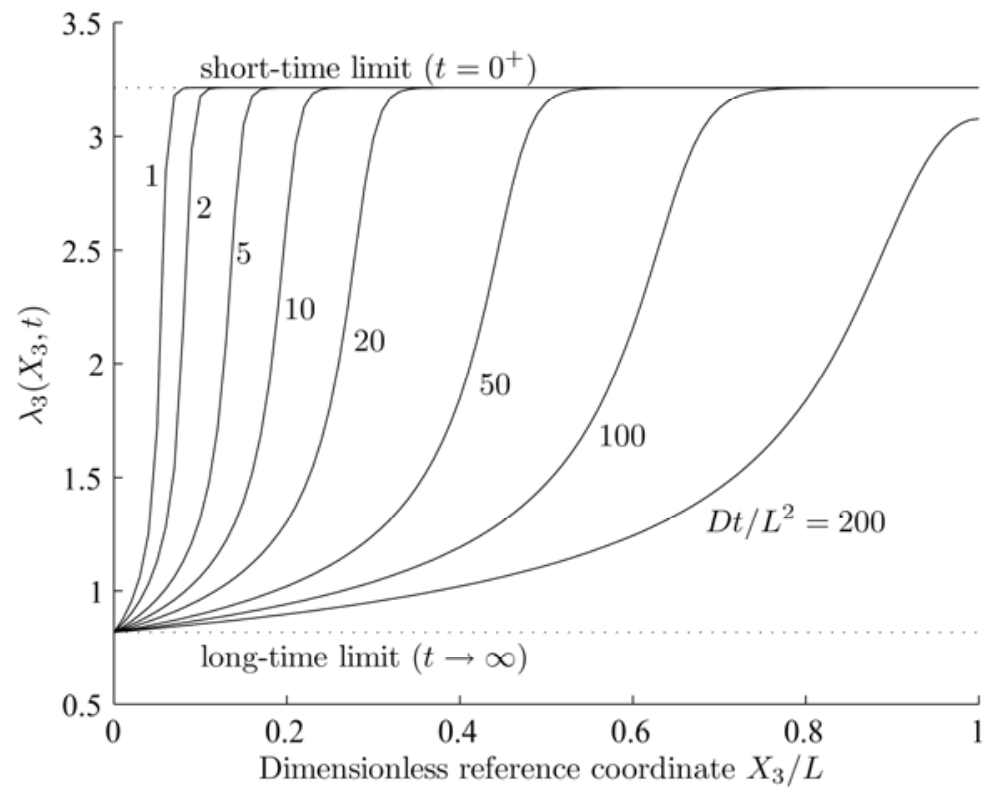

Figure 4. A gel $\left(N v=10^{-3}, \chi=0.2\right)$ is subject to a nominal stress $v s / k T=-0.05$. The stretch $\lambda_{3}$ is inhomogeneous and evolves from the short-time limit to the long-time limit. The surface of the gel ( $X_{3}=0$ ) is taken to equilibrate with the solvent instantaneously, but the gel beneath the surface equilibrate gradually. 


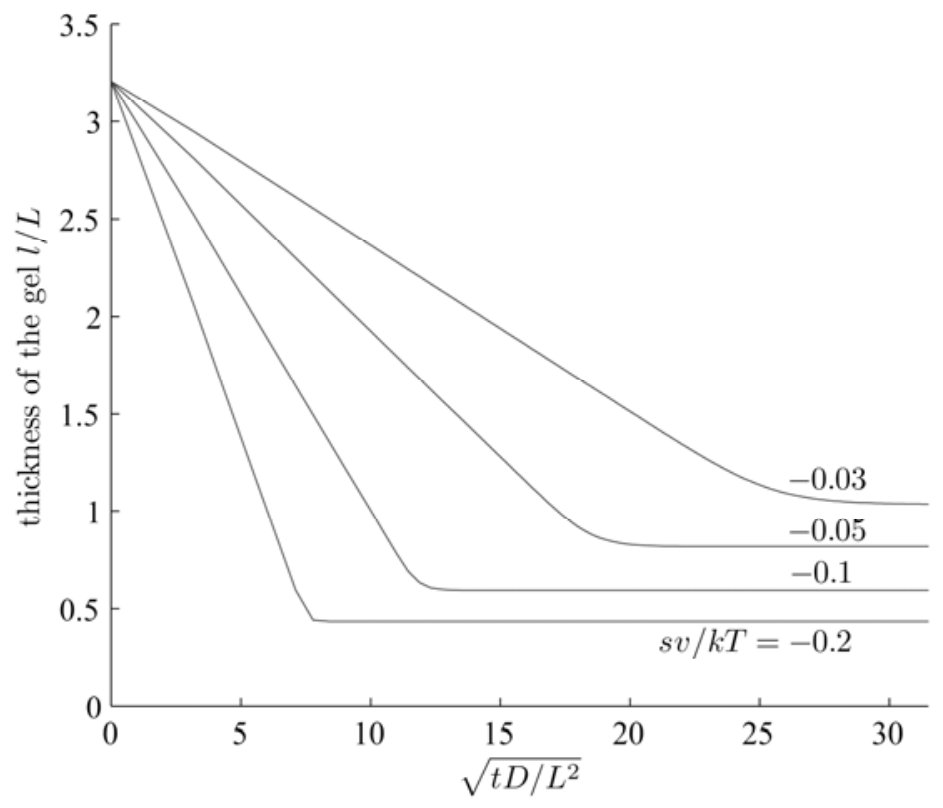

Figure 5. The thickness of the gel as a function of time. 


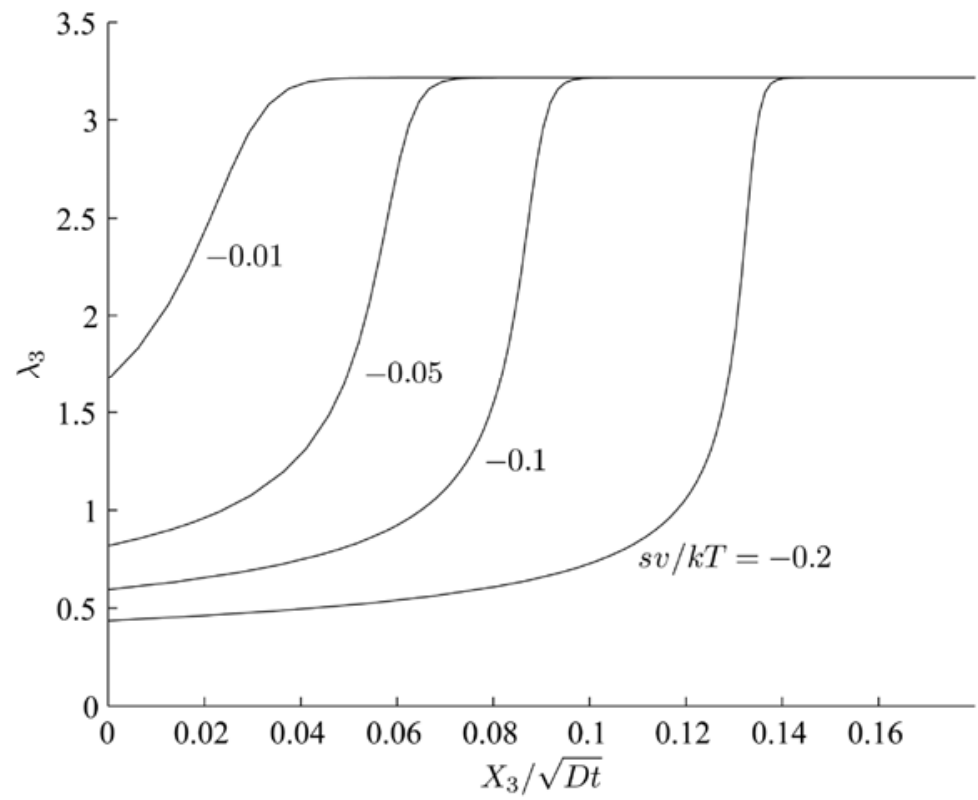

Figure 6. The self-similar solution of an infinitely thick gel. 

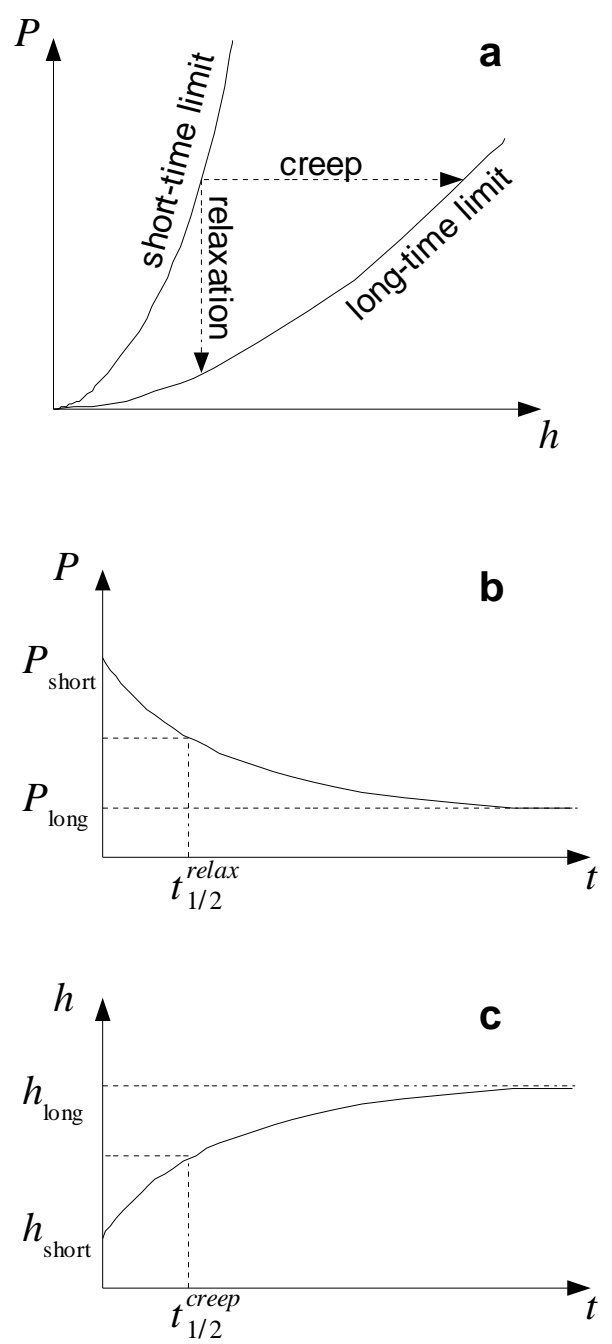

Figure 7. A conical indenter is suddenly pressed into the gel. (a) Both the long- and the short-time limit scale as $P \propto h^{2}$. A relaxation experiment corresponds to a vertical line, and a creep experiment corresponds to a horizontal line. (b) In a relaxation experiment, the depth $h$ is held constant, while the force $P$ decreases over time. (c) In a creep experiment, the force $P$ is held constant, while the depth $h$ increases over time. 